\title{
Identification of Pests on Plants using Clustering and Hybrid Approaches
}

\author{
Karkuzhali S, Krishna Mohan S , Kavin S , Karthick V I
}

\begin{abstract}
Enormous agricultural yield is lost each year, because of quick pervasion by pest and insects. A great deal of research is being done worldwide to recognize logical procedures for early discovery/identification of these bio-aggressors. In the past years, a few methodologies dependent on computerization and digital image processing have become known to address this issue. The greater part of the calculations focus on pest identification and location, restricted to a greenhouse environment. Likewise, they include a few complex computations to accomplish the equivalent. In this paper, we developed a unique algorithmic approach to isolate and distinguish pest utilizing clustering and hybrid approaches. The proposed method includes decreased computational complexity and pest detection in green house environment. The whitefly, a bio-aggressor which represents a risk to a huge number of harvests, was picked as the pest of enthusiasm for this paper. The calculation was tried for a few whiteflies influencing various leaves and an accuracy of $96 \%$ of whitefly recognition was accomplished.
\end{abstract} classification.

\section{INTRODUCTION}

$\mathrm{T}$ he primary focus of this paper is to use the highlights of advanced mobile phone and installed ideas to make farming straightforward. The objective of this paper is to draw out an effective instrument that lessens the exertion in keeping up agrarian fields and crops. Insects and irritations harm the yields and, consequently, are extremely hazardous for the general development of the harvest. One strategy to secure the harvest is early pest location with the goal that the yield can be shielded from pest attack. The most ideal approach to think about the wellbeing of the harvest is the convenient examination of the yield. In the event that nuisances are identified, suitable measures can be taken to shield the harvest from a major protection lost at the end. Early detection would be useful for limiting the utilization of the pesticides and would give direction to the selection of the pesticides.

Revised Manuscript Received on December 16, 2019.

* Correspondence Author

Karkuzhali S, Associate Professor, Department of Computer Science and Engineering from the Arulmigu Kalasalingam College of Engineering, Anna University, Chennai. India.

Krishna Mohan S , Associate Professor, Department of Computer Science and Engineering from the Arulmigu Kalasalingam College of Engineering, Anna University, Chennai. India.

Kavin S, Associate Professor, Department of Computer Science and Engineering from the Arulmigu Kalasalingam College of Engineering, Anna University, Chennai. India.

Karthick V I, Associate Professor, Department of Computer Science and Engineering from the Arulmigu Kalasalingam College of Engineering, Anna University, Chennai. India.
Keywords : Pest, clustering, hybrid approaches, segmentation,

\section{EXISTING METHOD}

Karen et al. (2018) presented a automatic pest recognition framework that applies artificial neural network systems. [1]. Bharthi et al. (2011) gives a propels in different strategies used to study plant ailments/attributes utilizing image processing. The techniques contemplated are for expanding throughput and lessening abstraction emerging from human specialists in identifying the plant disease [2].

Johnny et al. (2014) utilized the strategies of image analysis are widely connected to horticultural science, and it gives most extreme assurance to crops, which can at last lead to all the more likely yield the board and generation. Checking of pest pervasion depends on labor, anyway programmed observing has been progressing so as to limit human endeavors and mistakes [3]. Ganesh et al. (2013) depicts a product model framework for irritation recognition on the affected image of various leaves [4].

Preetha et al.(2016) utilized distinctive image processing methods for irritation location what's more, plant ailment identification. The affected images preparing procedure demonstrated as a successful machine vision framework for farming area [5]. Yi et al. (2018) proposed a novel superresoultion model dependent on deep recursive residual system. It accomplishes the best in existing method performance [6].

Pest acknowledgment and identification are crucial for sustenance security, a stable agrarian economy furthermore, personal satisfaction. To acknowledge fast discovery and acknowledgment of bug bugs, techniques enlivened by human visual framework were proposed by Deng et al.(2018) [7]. Ebrahimi et al.(2017) built up a technique to distinguish of thrips on the harvest shelter pictures utilizing SVM characterization strategys [8].

Zahid et al.(2018) presents a review on the various techniques pertinent to plants leaves of citrus fruit sicknesses recognition \& order. The article displays a point by point scientific categorization of citrus leaf sicknesses. At first, the difficulties of each progression are talked about in detail, which influences the recognition and arrangement precision. What's more, an intensive writing review of algorithms for segmentation and classification is introduced [9].

Murali et al. (2013) propose a unique methodology for early discovery of the of pest on harvests. To distinguish natural detection on an unpredictable foundation, we consolidated scanner image securing, examining streamlining, and progressed subjective vision. 
It shows the joint effort of reciprocal controls and methods, which prompted a computerized, powerful and flexible framework [10]. Yan Li et al. (2015) the recognition of little estimated irritations is most 28 attainable with the proposed multifractal investigation under nursery condition [11]. Preetha et al.(2016) proposed an automated pest distinguishing proof framework utilizing picture preparing methods. [12].

. The proposed RTBnet was a consequence of precise fitting and improvement to best in class onestage profound learning identifier RetinaNet. The key understanding behind the RTBnet configuration was to fit the identifier to the particular in-trap recognizable proof situation [13]. Yao et al. (2017) has built up a three-layer discovery strategy is plausible and compelling for the recognizable proof of various formative phases of planthoppers on rice plants in paddy fields [14].

\section{PROPOSED METHOD}

The data of affected leaf images are collected in agriculture field near the village Watrap. Clustering is an iterative method that is utilized to divide a image into groups based on the similarity. Methodology of grouping technique. Groups can be chosen physically, arbitrarily, or dependent on certain conditions. Separation between the pixel and group focus is determined by the squared or supreme contrast between a pixel and a bunch focus. The segmentation of images alone can't give great quality yield, it needs preprocessing step. Preprocessing may comprise of different advances like de-noising and image enhancement. De-noising gave the rank channel, which appropriate for the vermin picture. It is non-straight channel, which protect the shape, edge and other data without absence of lucidity. Because of the abnormalities and downsides in pestimages, it is obligatory to incorporate the preprocessing venture before the image segmentation for the quality and precise yield. The figure 1 shows the block diagram of the proposed method. The figure 2 and 3 shows the implementation result of the proposed method.

\section{CONCLUSION}

The utilization of artificial pest sprays and nematicides have been debasing the nature of ranch crops for a long time. In this paper a novel calculation is introduced for effectively distinguishing the bug tainted regions of these yields. The calculation can be additionally changed for finding the ailing territories in the harvests by utilizing modern programming projects and better picture securing devices. All over the world farming specialists are working one radication of bioagressors and contaminated espresso estate are one of the difficulties out of it. Picture Processing strategy assumes a fundamental job in it. Our first target is to distinguish sicknesses like espresso berry on estates and other bio agressors (aphids) or plant infections. Intellectual methodologies introduce new articles to distinguish or new image processing programs also remove the comparing data. and potato crops by applying neural classifiers. Engineering in Agriculture, Environment and Food, 11(4), pp.245-255.

[2] Patil, J.K. and Kumar, R., 2011. Advances in image processing for detection of plant diseases. Journal of Advanced Bioinformatics Applications and Research, 2(2), pp.135-141

[3] Miranda, J.L., Gerardo, B.D. and Tanguilig III, B.T., 2014. Pest detection and extraction using image processing techniques. International Journal of Computer and Communication Engineering, 3(3), p.189.

[4] Bhadane, G., Sharma, S. and Nerkar, V.B., 2013. Early pest identification in agricultural crops using image processing techniques. International Journal of Electrical, Electronics and Computer Engineering, 2(2), pp.77-82.

[5] Rajan, P. and Radhakrishnan, B., 2016. A Survey on Different Image Processing Techniques for Pest Identification and Plant Disease Detection. International Journal of Computer Science and Network, 5(1), pp.137-141.

[6] Yue, Y., Cheng, X., Zhang, D., Wu, Y., Zhao, Y., Chen, Y., Fan, G. and Zhang, Y., 2018. Deep recursive super resolution network with Laplacian Pyramid for better agricultural pest surveillance and detection. Computers and electronics in agriculture, 150, pp.26-32.

[7] Deng, L., Wang, Y., Han, Z. and Yu, R., 2018. Research on insect pest image detection and recognition based on bio-inspired methods. Biosystems Engineering, 169, pp.139-148.

[8] Ebrahimi, M.A., Khoshtaghaza, M.H., Minaei, S. and Jamshidi, B., 2017. Vision-based pest detection based on SVM classification method. Computers and Electronics in Agriculture, 137, pp.52-58.

[9] Iqbal, Z., Khan, M.A., Sharif, M., Shah, J.H., ur Rehman, M.H. and Javed, K., 2018. An automated detection and classification of citrus plant diseases using image processing techniques: A review. Computers and electronics in agriculture, 153, pp.12-32.

[10] Krishnan, M. and Jabert, G., 2013. Pest Control in Agricultural Plantations Using Image Processing. IOSR Journal of Electronics and Communication Engineering (IOSR-JECE), 6(4), pp.68-74.

[11] Li, Y., Xia, C. and Lee, J., 2015. Detection of small-sized insect pest in greenhouses based on multifractal analysis. Optik-International Journal for Light and Electron Optics, 126(19), pp.2138-2143.

[12] Rajan, P., Radhakrishnan, B. and Suresh, L.P., 2016, October. Detection and classification of pests from crop images using Support Vector Machine. In 2016 international conference on emerging technological trends (ICETT) (pp. 1-6). IEEE.

[13] Sun, Y., Liu, X., Yuan, M., Ren, L., Wang, J. and Chen, Z., 2018. Automatic in-trap pest detection using deep learning for pheromone-based Dendroctonus valens monitoring. Biosystems Engineering, 176, pp.140-150.

[14] Qing, Y.A.O., CHEN, G.T., Zheng, W.A.N.G., ZHANG, C., YANG, B.J. and Jian, T.A.N.G., 2017. Automated detection and identification of white-backed planthoppers in paddy fields using image processing. Journal of integrative agriculture, 16(7), pp.1547-1557.

\section{AUTHORS PROFILE}

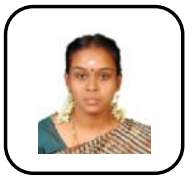

Dr. S.Karkuzhali received the B.E degree in Computer Science and Engineering from the Arulmigu Kalasalingam College of Engineering affiliated to Anna University, Chennai in 2008, and the M.E. degree in Computer and Communication Engineering from the National Engineering College affiliated to Anna University of Technology Tirunelveli, in 2011.She secured first rank in her M.E degree under those colleges which are affiliated under Anna University of Technology Tirunelveli. She completed her PhD (Information and Communication Engineering) and the thesis entitled "Analysis of retinal images for diagnosis of Eye Diseases using Feature Extraction" in Anna University, Chennai. She is currently working as Associate Professor in Kalasalingam Academy of Research and Education. Her research interests are in the areas of Medical image processing, Computer vision, Pattern Recognition, and Soft Computing techniques. She had published more than thirty papers in National, International Journals and Conferences

\section{REFERENCES}

[1] Roldán-Serrato, K.L., Escalante-Estrada, J.A.S. and Rodríguez-González, M.T., 2018. Automatic pest detection on bean 


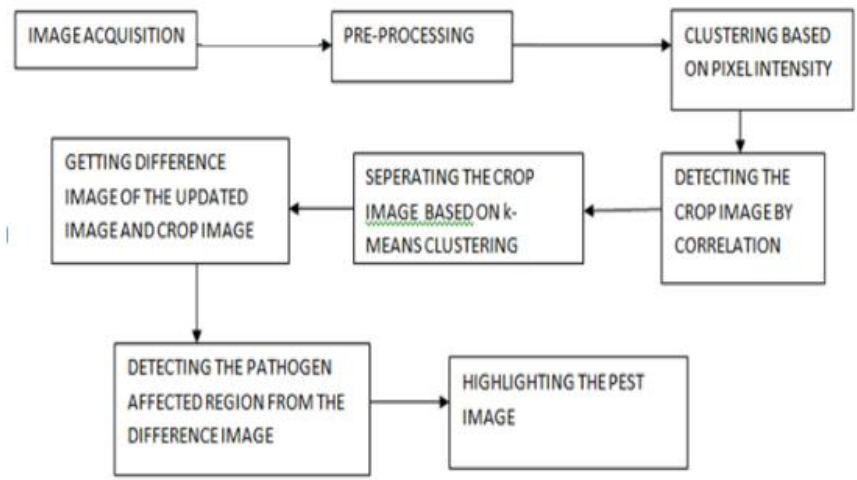

Figure 1 : Block Diagram of the Proposed Method of Identification of Pests

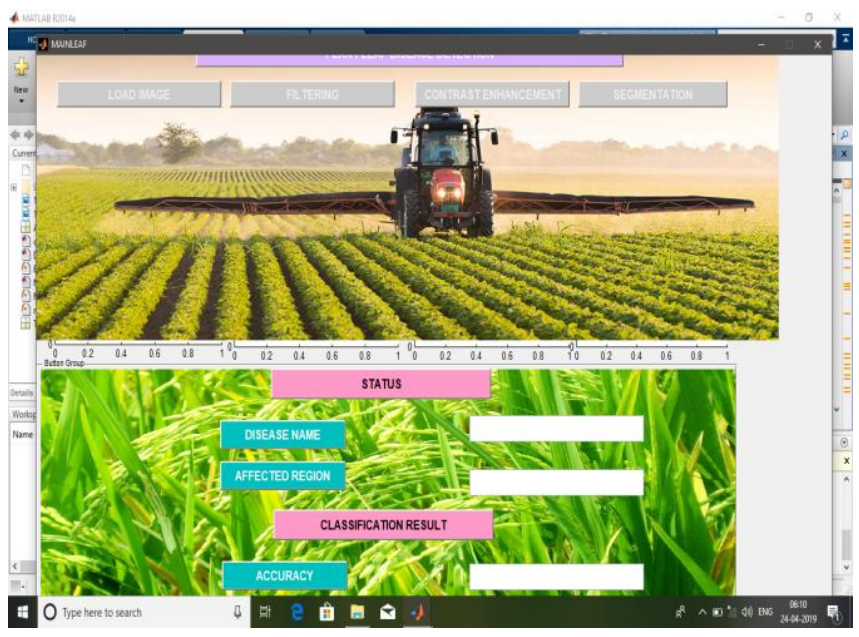

Figure 2 :Implementation Result of the proposed Method

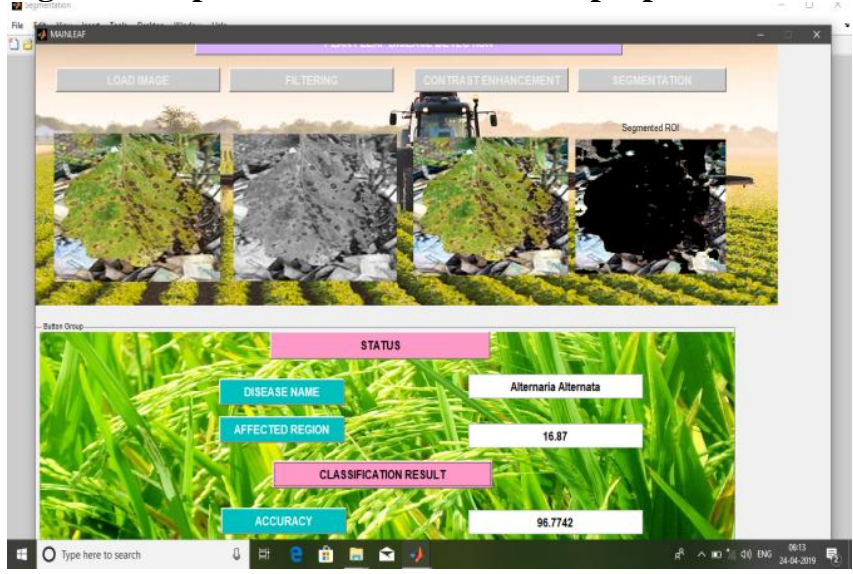

Figure 3: Sample output of the Proposed Method 